\title{
Heavy flavor spectroscopy results from CMS
}

\section{Sergey Polikarpov*t}

Lebedev Physical Institute of the Russian Academy of Sciences (LPI RAS), Moscow, Russia,

E-mail: sergey.polikarpovecern.ch

The observation of the $\mathrm{B}_{\mathrm{s} 2}^{*}(5840)^{0} \rightarrow \mathrm{B}^{0} \mathrm{~K}_{S}^{0}$ decay and the evidence for the $\mathrm{B}_{\mathrm{s} 1}(5830)^{0} \rightarrow \mathrm{B}^{* 0} \mathrm{~K}_{S}^{0}$ decay by the CMS experiment are reported, as well as the study of the $\mathrm{B}^{+} \rightarrow \mathrm{J} / \psi \bar{\Lambda} \mathrm{p}$ decay, performed using the data collected in proton-proton collisions at $\sqrt{s}=8 \mathrm{TeV}$. In addition, the observation of the $\Lambda_{\mathrm{b}}^{0} \rightarrow \mathrm{J} / \psi \Lambda \phi$ decay and measurement of its branching fraction, relative to the $\Lambda_{\mathrm{b}}^{0} \rightarrow \psi(2 \mathrm{~S}) \Lambda$ decay, using the proton-proton collision data collected at at $\sqrt{s}=13 \mathrm{TeV}$ is presented.

18th International Conference on B-Physics at Frontier Machines - Beauty2019 -

29 September $/ 4$ October, 2019

Ljubljana, Slovenia

* Speaker.

${ }^{\dagger}$ on behalf of the CMS Collaboration 
The CMS experiment [1] at the LHC is providing new important results in the Heavy Flavor physics sector. In this work, we report the study of excited $\mathrm{B}_{\mathrm{s}}^{0}$ mesons [2], including the first observation of the $\mathrm{B}_{\mathrm{s} 2}^{*} \rightarrow \mathrm{B}^{0} \mathrm{~K}_{S}^{0}$ decay and the measurements of $\mathrm{B}_{\mathrm{s} 2}^{*}$ and $\mathrm{B}_{\mathrm{s} 1}$ meson properties, the study of $\mathrm{B}^{+} \rightarrow \mathrm{J} / \psi \bar{\Lambda} \mathrm{p}$ decay including the intermediate resonance study [3], and the first observation of the $\Lambda_{\mathrm{b}}^{0} \rightarrow \mathrm{J} / \psi \Lambda \phi$ decay [4]. Charge-conjugate states are implied throughout the text.

\section{Study of P-wave $B_{s}^{0}$ states}

Experimental information about the excited $\mathrm{B}_{\mathrm{s}}^{0}$ mesons is very limited. In particular, the $P$ wave $\mathrm{B}_{\mathrm{s}}^{0}$ mesons were observed by the $\mathrm{CDF}$ and $\mathrm{D} 0$ collaborations at the Tevatron $[5,6]$ as narrow peaks in the $\mathrm{B}^{+} \mathrm{K}^{-}$invariant mass distribution. Later, the $\mathrm{LHCb}$ collaboration at the LHC presented precise measurements of the $\mathrm{B}_{\mathrm{s} 1,2}^{(*)}$ properties [7], including the observation of the $\mathrm{B}_{\mathrm{s} 2}^{*} \rightarrow \mathrm{B}^{*+} \mathrm{K}^{-}$ decay. More recently the CDF collaboration released a study of orbitally excited B mesons [8], which included updated measurements of $\mathrm{B}_{\mathrm{s} 1,2}^{(*)}$ properties. All these analyses used only the decays into charged $\mathrm{B}$ meson and a kaon to reconstruct the $\mathrm{B}_{\mathrm{s} 1,2}^{(*)}$ candidates, while we report on the search result for the decays into a neutral $\mathrm{B}$ meson and a neutral kaon.

Using the data sample collected by the CMS experiment in proton-proton collisions at $\sqrt{s}=$ $8 \mathrm{TeV}$, corresponding to an integrated luminosity of about $20 \mathrm{fb}^{-1}$, we reconstruct the $\mathrm{B}^{+} \mathrm{K}^{-}$and $\mathrm{B}^{0} \mathrm{~K}_{S}^{0}$ candidates. The $\mathrm{B}^{+}$and $\mathrm{B}^{0}$ mesons are reconstructed using the decays $\mathrm{B}^{+} \rightarrow \mathrm{J} / \psi \mathrm{K}^{+}$and $\mathrm{B}^{0} \rightarrow \mathrm{J} / \psi \mathrm{K}^{* 0}\left(\mathrm{~K}^{* 0} \rightarrow \mathrm{K}^{+} \pi^{-}\right)$, where $\mathrm{J} / \psi \rightarrow \mu^{+} \mu^{-}$, and the two muons trigger the event readout. Standard requirements are applied on the muon and track quality and muon identification. The $\mathrm{B}$ meson vertices are required to be significantly displaced from the pp interaction vertex and the $\mathrm{B}$ meson momentum is required to be collinear with the direction from the pp interaction vertex to the $\mathrm{B}$ meson vertex. The $\mathrm{K}_{S}^{0}$ candidates are selected from the collection of two-track vertices significantly displaced from the pp interaction region, as described in Ref. [9].
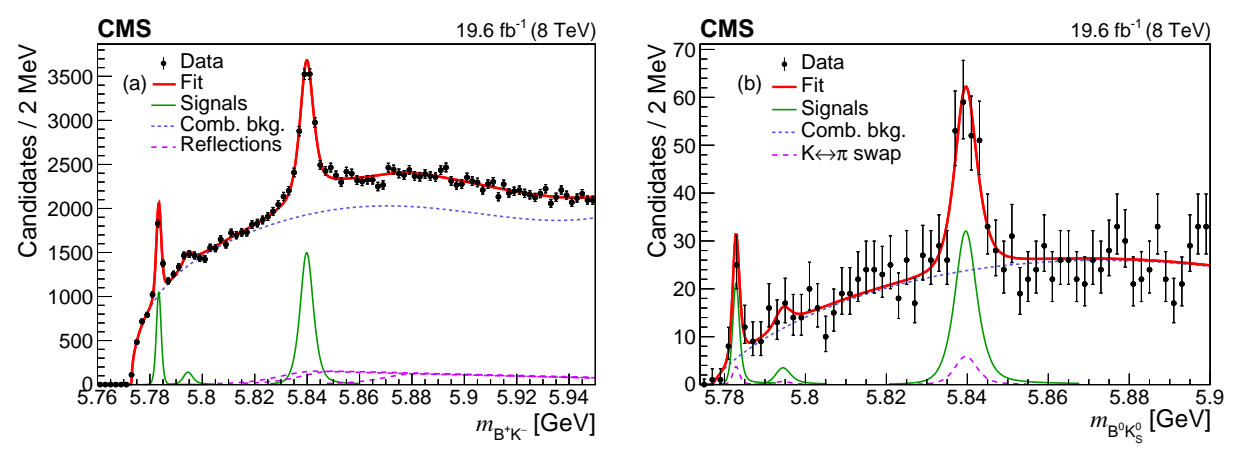

Figure 1: The $\mathrm{B}^{+} \mathrm{K}^{-}$(left) and $\mathrm{B}^{0} \mathrm{~K}_{S}^{0}$ (right) invariant mass distributions in data [2].

Figure 1 (left) shows the invariant mass distribution of the selected $\mathrm{B}^{+} \mathrm{K}^{-}$candidates with the fit results overlaid. The background model includes the smooth combinatorial background component and the three components accounting for the contributions from the $\mathrm{B}_{2}^{*}(5747)^{0} \rightarrow \mathrm{B}^{+} \pi^{-}$, $\mathrm{B}_{2}^{*}(5747)^{0} \rightarrow \mathrm{B}^{*+} \pi^{-}$, and $\mathrm{B}_{1}(5721)^{0} \rightarrow \mathrm{B}^{*+} \pi^{-}$decays, with shapes obtained in the simulation. The three signals, corresponding to the decays $\mathrm{B}_{\mathrm{s} 2}^{*} \rightarrow \mathrm{B}^{+} \mathrm{K}^{-}, \mathrm{B}_{\mathrm{s} 2}^{*} \rightarrow \mathrm{B}^{*+} \mathrm{K}^{-}$, and $\mathrm{B}_{\mathrm{s} 1} \rightarrow \mathrm{B}^{*+} \mathrm{K}^{-}$, are modelled with relativistic Breit-Wigner functions convolved with the resolution. 
Figure 1 (right) shows the measured $m_{\mathrm{B}^{0} \mathrm{~K}_{S}^{0}}$ distribution. Similar to the $\mathrm{B}^{+} \mathrm{K}^{-}$channel, the three signal functions are included accounting for the $\mathrm{B}_{\mathrm{s} 2}^{*} \rightarrow \mathrm{B}^{0} \mathrm{~K}_{S}^{0}, \mathrm{~B}_{\mathrm{s} 2}^{*} \rightarrow \mathrm{B}^{* 0} \mathrm{~K}_{S}^{0}$, and $\mathrm{B}_{\mathrm{s} 1} \rightarrow$ $\mathrm{B}^{* 0} \mathrm{~K}_{S}^{0}$ decays. The charged pion and kaon may be swapped in the $\mathrm{B}^{0} \rightarrow \mathrm{J} / \psi \mathrm{K}^{+} \pi^{-}$reconstruction, which leads to narrow peaks at the same $m_{\mathrm{B}^{0} \mathrm{~K}_{S}^{0}}$ value, as found in simulation; the corresponding contributions are included in the fit model.

The unbinned maximum-likelihood (UML) fit results and efficiencies from the simulation are used to measure the relative branching fractions [2]

$$
\begin{aligned}
& R_{2}^{0 \pm}=\frac{\mathscr{B}\left(\mathrm{B}_{\mathrm{s} 2}^{*} \rightarrow \mathrm{B}^{0} \mathrm{~K}_{S}^{0}\right)}{\mathscr{B}\left(\mathrm{B}_{\mathrm{s} 2}^{*} \rightarrow \mathrm{B}^{+} \mathrm{K}^{-}\right)}=0.432 \pm 0.077 \text { (stat) } \pm 0.075 \text { (syst) } \pm 0.021(\mathrm{PDG}) \\
& R_{1}^{0 \pm}=\frac{\mathscr{B}\left(\mathrm{B}_{\mathrm{s} 1} \rightarrow \mathrm{B}^{* 0} \mathrm{~K}_{S}^{0}\right)}{\mathscr{B}\left(\mathrm{B}_{\mathrm{s} 1} \rightarrow \mathrm{B}^{*+} \mathrm{K}^{-}\right)}=0.492 \pm 0.122 \text { (stat) } \pm 0.068 \text { (syst) } \pm 0.024(\mathrm{PDG}) \\
& R_{2 *}^{ \pm}=\frac{\mathscr{B}\left(\mathrm{B}_{\mathrm{s} 2}^{*} \rightarrow \mathrm{B}^{*+} \mathrm{K}^{-}\right)}{\mathscr{B}\left(\mathrm{B}_{\mathrm{s} 2}^{*} \rightarrow \mathrm{B}^{+} \mathrm{K}^{-}\right)}=0.081 \pm 0.021 \text { (stat) } \pm 0.015 \text { (syst) } \\
& R_{2 *}^{0}=\frac{\mathscr{B}\left(\mathrm{B}_{\mathrm{s} 2}^{*} \rightarrow \mathrm{B}^{* 0} \mathrm{~K}_{S}^{0}\right)}{\mathscr{B}\left(\mathrm{B}_{\mathrm{s} 2}^{*} \rightarrow \mathrm{B}^{0} \mathrm{~K}_{S}^{0}\right)}=0.093 \pm 0.086 \text { (stat) } \pm 0.014 \text { (syst)}
\end{aligned}
$$

where the first uncertainties are statistical, the second systematic, and the third are due to the uncertainties in the branching fractions of the intermediate decays. The dominant systematic uncertainties in these measurements are related to the choice of the fit model and the track reconstruction efficiency uncertainty.

The $\mathrm{B}_{\mathrm{s} 2}^{*}(5840)^{0}$ natural width is determined to be $1.52 \pm 0.34$ (stat) \pm 0.30 (syst) $\mathrm{MeV}$ [2]. The mass differences measured for the first time are [2]

$$
\begin{aligned}
M\left(\mathrm{~B}_{\mathrm{s} 2}^{*}\right)-M\left(\mathrm{~B}^{0}\right)-M\left(\mathrm{~K}_{S}^{0}\right) & =62.37 \pm 0.48(\text { stat }) \pm 0.07(\text { syst }) \mathrm{MeV} \\
M\left(\mathrm{~B}_{\mathrm{s} 1}\right)-M\left(\mathrm{~B}^{* 0}\right)-M\left(\mathrm{~K}_{S}^{0}\right) & =5.61 \pm 0.23(\text { stat }) \pm 0.06(\text { syst }) \mathrm{MeV} \\
M\left(\mathrm{~B}^{* 0}\right)-M\left(\mathrm{~B}^{*+}\right) & =0.91 \pm 0.24(\text { stat }) \pm 0.09(\text { syst }) \pm 0.02(\mathrm{PDG}) \mathrm{MeV}
\end{aligned}
$$

\section{Study of the $\mathrm{B}^{+} \rightarrow \mathrm{J} / \psi \bar{\Lambda} \mathrm{p}$ decay}

The multibody decays of heavy hadrons involving a charmonium state and a baryon in the decay products provide an excellent laboratory to search for exotic states decaying into charmonium and a baryon, i.e. pentaquark candidates. Such states have been observed by the LHCb collaboration by studying the $\mathrm{J} / \psi \mathrm{p}$ mass distribution from the $\Lambda_{\mathrm{b}}^{0} \rightarrow \mathrm{J} / \psi \mathrm{pK}^{-}$decay [10]. The decay $\mathrm{B}^{+} \rightarrow \mathrm{J} / \psi \bar{\Lambda}$ p provides an opportunity to study the intermediate $\mathrm{J} / \psi \mathrm{p}$ and $\mathrm{J} / \psi \bar{\Lambda}$ mass distributions.

This decay is studied using the pp collision data collected by the CMS detector in 2012, using the $\mathrm{J} / \psi \rightarrow \mu^{+} \mu^{-}$and $\Lambda \rightarrow \mathrm{p} \pi^{-}$decays to reconstruct $\mathrm{B}^{+}$candidates. The observed invariant mass distribution, presented in Fig. 2, shows a clean peak at the $\mathrm{B}^{+}$mass on top of relatively low background. The signal yield, measured with an UML fit, us $452 \pm 23$ [3]. The similar in topology $\mathrm{B}^{+} \rightarrow \mathrm{J} / \psi \mathrm{K}^{*}(892)^{+}\left(\mathrm{K}^{*}(892)^{+} \rightarrow \mathrm{K}_{S}^{0} \pi^{+}\right)$decay is used for the normalization to perform a measurement of the branching fraction ratio

$$
\frac{\mathscr{B}\left(\mathrm{B}^{+} \rightarrow \mathrm{J} / \psi \bar{\Lambda} \mathrm{p}\right)}{\mathscr{B}\left(\mathrm{B}^{+} \rightarrow \mathrm{J} / \psi \mathrm{K}^{*}(892)^{+}\right)}=(1.054 \pm 0.057(\text { stat }) \pm 0.035(\text { syst }) \pm 0.011(\mathrm{PDG})) \%
$$




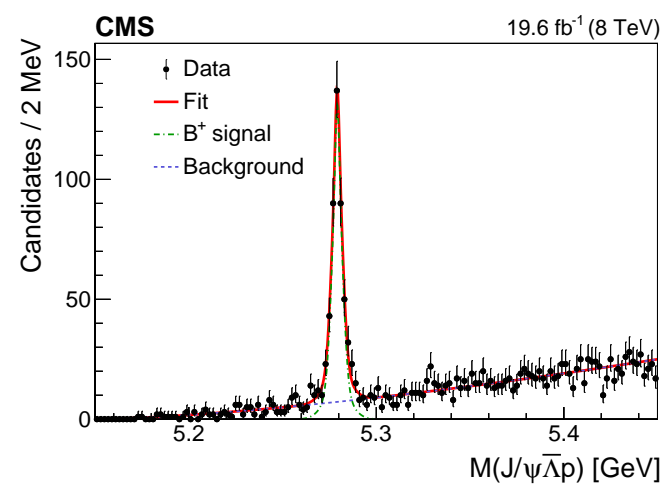

Figure 2: The $\mathrm{J} / \psi \bar{\Lambda} \mathrm{p}$ invariant mass distribution [3].

where the last uncertainty is related to the knowledge of the branching fractions of the intermediate decays involved. This measurement allows to obtain $\mathscr{B}\left(\mathrm{B}^{+} \rightarrow \mathrm{J} / \psi \bar{\Lambda} \mathrm{p}\right)=(15.1 \pm 0.8 \pm 0.5 \pm 0.9) \times$ $10^{-6}[3]$, which is the most precise measurement to date.
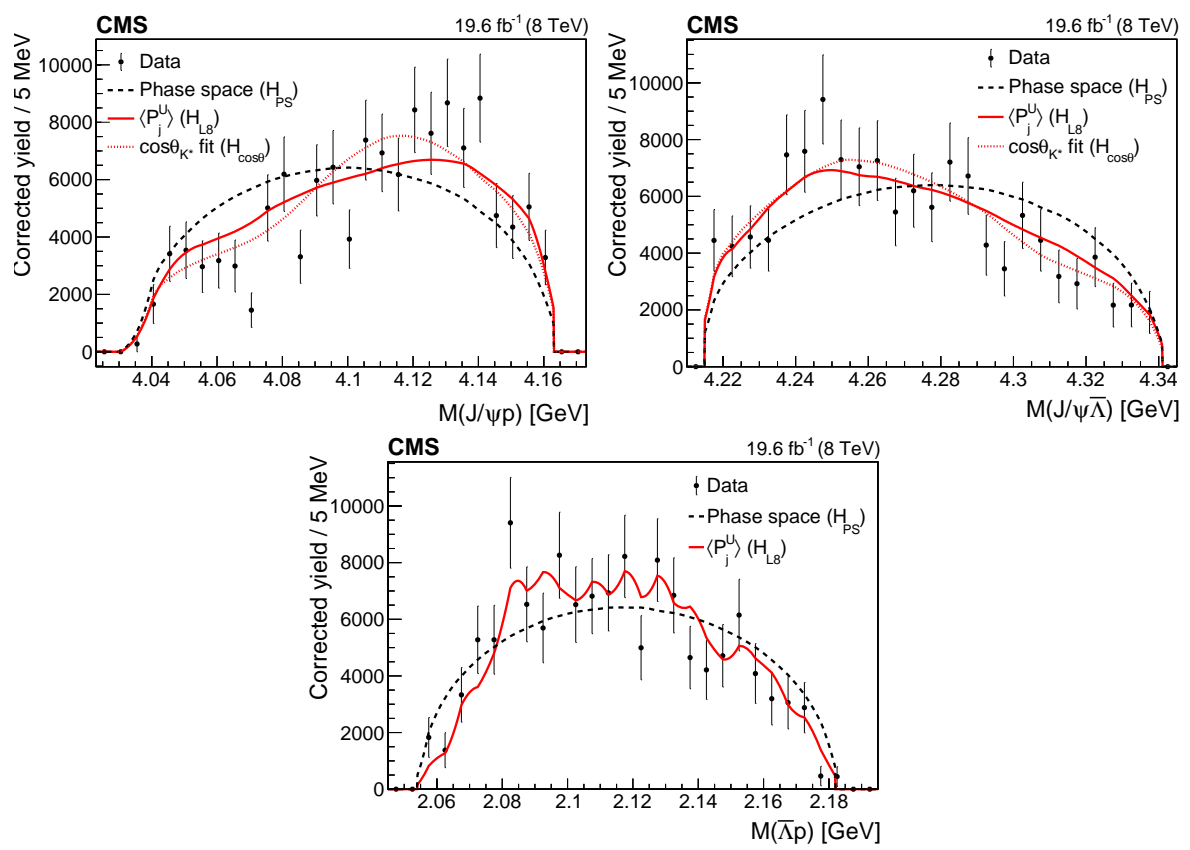

Figure 3: The efficiency-corrected, background-subtracted $\mathrm{J} / \psi \mathrm{p}$ (left), $\mathrm{J} / \psi \bar{\Lambda}$ (right), and $\bar{\Lambda} \mathrm{p}$ (bottom) invariant mass distributions in the $\mathrm{B}^{+} \rightarrow \mathrm{J} / \psi \bar{\Lambda} \mathrm{p}$ decay. The dashed lines correspond to phase space fit, the solid lines show the phase space distribution corrected for the $\bar{\Lambda}$ p angular structure accounting for resonances with spin up to 4 , and the dotted curves illustrate the phase space distributions reweighted according to the $\cos \left(\theta_{\mathrm{K}^{*}}\right)$ distribution [3].

Additionally, the $\mathrm{J} / \psi \mathrm{p}, \mathrm{J} / \psi \bar{\Lambda}$, and $\bar{\Lambda} \mathrm{p}$ mass distributions from the $\mathrm{B}^{+} \rightarrow \mathrm{J} / \psi \bar{\Lambda} \mathrm{p}$ decays are investigated. Firstly, they are background-subtacted using the $\mathrm{B}^{+}$mass fit mentioned above, and efficiency-corrected using the efficiency, as a function of $\mathrm{p} \bar{\Lambda}$ mass and angular parameter $\cos \left(\theta_{\mathrm{K}^{*}}\right)$, 
obtained in the simulated samples. The $\mathrm{J} / \psi \mathrm{p}, \mathrm{J} / \psi \bar{\Lambda}$, and $\bar{\Lambda} \mathrm{p}$ mass distributions are found to be inconsistent with pure 3-body phase-space $\mathrm{B}^{+} \rightarrow \mathrm{J} / \psi \bar{\Lambda}$ p decay hypothesis. To account for possible contributions from the excited kaon decays into $\bar{\Lambda}$, a model-independent approach is utilized [3]. As a result, the consistency between the data and the model is significantly improved after accounting for possible contributions from excited kaons with spin up to 4, as shown in Fig. 3 with the solid line, and the level of remaining inconsistency is estimated to be below $2.8 \sigma$.

\section{Observation of the $\Lambda_{\mathrm{b}}^{0} \rightarrow \mathrm{J} / \psi \Lambda \phi$ decay}

Another example of a b hadron decay that allows to study intermediate resonances in $\mathrm{J} / \psi+$ baryon spectrum is the $\Lambda_{\mathrm{b}}^{0} \rightarrow \mathrm{J} / \psi \Lambda \phi$ decay. It is searched for using the $13 \mathrm{TeV}$ pp collision data collected with the CMS experiment in 2018. The candidates are reconstructed using the $\mathrm{J} / \psi \rightarrow$ $\mu^{+} \mu^{-}, \phi \rightarrow \mathrm{K}^{+} \mathrm{K}^{-}$, and $\Lambda \rightarrow \mathrm{p} \pi^{-}$decays for the search channel; the decay $\psi(2 \mathrm{~S}) \rightarrow \mathrm{J} / \psi \pi^{+} \pi^{-}$is used in the reconstruction of the normalization channel $\Lambda_{\mathrm{b}}^{0} \rightarrow \psi(2 \mathrm{~S}) \Lambda$. Signal and normalization channels have the same number of tracks in the final state, thus reducing systematic uncertainties.

Observed $\mathrm{J} / \psi \Lambda \phi$ invariant mass distribution is shown in Fig. 4, where the clearly visible signal is modelled with a double-Gaussian and the smooth background with a third-order polynomial function [4].
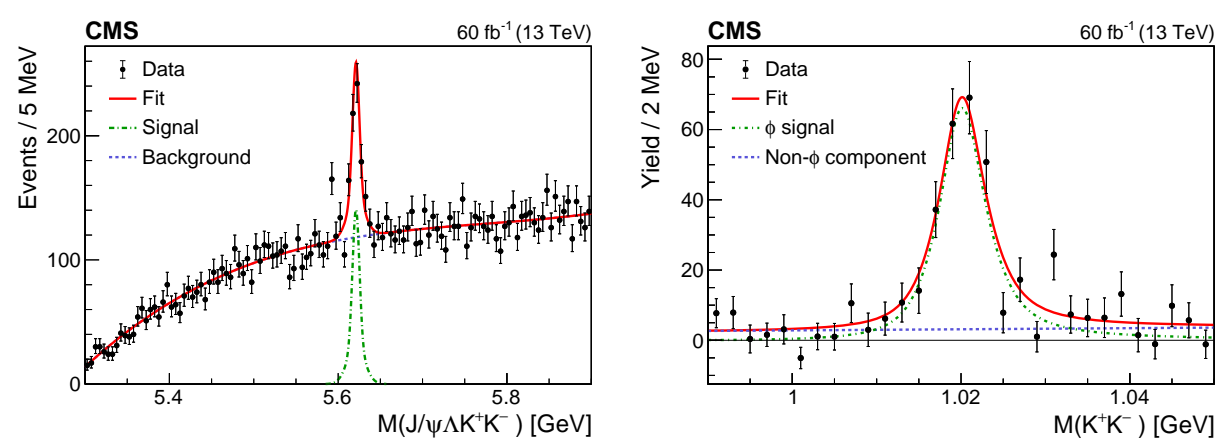

Figure 4: The $\mathrm{J} / \psi \Lambda \phi$ invariant mass distribution in data (left). Background-subtracted $\mathrm{K}^{+} \mathrm{K}^{-}$invariant mass distribution (right) [4].

The number of signal events is $380 \pm 32$ and the statistical significance is about $10 \sigma$ in the asymptotic approximation. The fit is used to obtain background-subtracted $M\left(\mathrm{~K}^{+} \mathrm{K}^{-}\right)$distribution shown in Fig 4 (right), which is subsequently fit with a sum of smooth background function and Breit-Wigner convolved with the resolution for $\phi$ signal. The number of signal $\phi$ events returned by this fit, $286 \pm 29$, is used together with the number of the reconstructed events in the normalization channel $\Lambda_{\mathrm{b}}^{0} \rightarrow \psi(2 \mathrm{~S}) \Lambda(884 \pm 37)$ and the relative efficiency from simulation to measure [4]

$$
\frac{\mathscr{B}\left(\Lambda_{\mathrm{b}}^{0} \rightarrow \mathrm{J} / \psi \Lambda \phi\right)}{\mathscr{B}\left(\Lambda_{\mathrm{b}}^{0} \rightarrow \psi(2 \mathrm{~S}) \Lambda\right)}=8.26 \pm 0.90(\text { stat }) \pm 0.68 \text { (syst) } \pm 0.11(\mathrm{PDG}) \%
$$

where the last uncertainty is related to the known branching fractions of the decays $\psi(2 \mathrm{~S}) \rightarrow$ $\mathrm{J} / \psi \pi^{+} \pi^{-}$and $\phi \rightarrow \mathrm{K}^{+} \mathrm{K}^{-}$. 


\section{Summary}

In summary, the $\mathrm{B}_{\mathrm{s} 2}^{*}(5840)^{0} \rightarrow \mathrm{B}^{0} \mathrm{~K}_{S}^{0}$ decay is observed for the first time, the evidence for the $\mathrm{B}_{\mathrm{s} 1}(5830)^{0} \rightarrow \mathrm{B}^{* 0} \mathrm{~K}_{S}^{0}$ decay is found, and the mass difference $M\left(\mathrm{~B}^{* 0}\right)-M\left(\mathrm{~B}^{*+}\right)$ is measured for the first time. The $\mathrm{B}^{+} \rightarrow \mathrm{J} / \psi \bar{\Lambda}$ p decay branching fraction is reported, and no evidence is found for exotic intermediate resonance contributions in this decay. These analyses were performed using the data sample of about $20 \mathrm{fb}^{-1}$ collected by the CMS experiment at the LHC in proton-proton collisions at $\sqrt{s}=8 \mathrm{TeV}$. In addition, the decay $\Lambda_{\mathrm{b}}^{0} \rightarrow \mathrm{J} / \psi \Lambda \phi$ is observed and its branching fraction is measured with respect to $\mathscr{B}\left(\Lambda_{\mathrm{b}}^{0} \rightarrow \psi(2 \mathrm{~S}) \Lambda\right)$ using the $\sqrt{s}=13 \mathrm{TeV}$ pp collision data.

\section{Acknowledgments}

The author's work was supported by the programme of the presidium of the Russian Academy of Sciences (No. 3 "Physics of fundamental interactions and nuclear technology", 2018-2020) and the megagrant 14.W03.31.0026 of the Ministry of Science and Education of Russian Federation. The author would like to thank the Beauty 2019 conference organizers for the excellent program, warm hospitality, and opportunity to present these important results.

\section{References}

[1] The CMS Collaboration, The CMS experiment at the CERN LHC, JINST 3 (2008) S08004.

[2] The CMS Collaboration, Studies of $\mathrm{B}_{\mathrm{s} 2}^{*}(5840)^{0}$ and $\mathrm{B}_{\mathrm{s} 1}(5830)^{0}$ mesons including the observation of the $\mathrm{B}_{\mathrm{s} 2}^{*}(5840)^{0} \rightarrow \mathrm{B}^{0} \mathrm{~K}_{S}^{0}$ decay in proton-proton collisions at $\sqrt{s}=8 \mathrm{TeV}$, Eur. Phys. J. C 78 (2018) 939.

[3] The CMS Collaboration, Study of the $\mathrm{B}^{+} \rightarrow \mathrm{J} / \psi \bar{\Lambda} \mathrm{p}$ decay in proton-proton collisions at $\sqrt{s}=8 \mathrm{TeV}$, arXiv: 1907.05461, accepted for publication in J. High Energy Phys.

[4] The CMS Collaboration, Observation of the $\Lambda_{\mathrm{b}}^{0} \rightarrow \mathrm{J} / \psi \Lambda \phi$ decay in proton-proton collisions at $\sqrt{s}=13 \mathrm{TeV}$, arXiv:1911.03789, submitted to Phys. Lett. B.

[5] The CDF Collaboration, Observation of orbitally excited $\mathrm{B}_{\mathrm{s}}$ mesons, Phys. Rev. Lett 100 (2008) 082001.

[6] The D0 Collaboration, Observation and properties of the orbitally excited $\mathrm{B}_{s 2}^{*}$ meson, Phys. Rev. Lett 100 (2008) 082002.

[7] The LHCb Collaboration, First observation of the decay $\mathrm{B}_{\mathrm{s} 2}^{*}(5840)^{0} \rightarrow \mathrm{B}^{*+} \mathrm{K}^{-}$and studies of excited $\mathrm{B}_{\mathrm{s}}^{0}$ mesons, Phys. Rev. Lett 110 (2013) 151803.

[8] The CDF Collaboration, Study of orbitally excited B mesons and evidence for a new $\mathrm{B} \pi$ resonance, Phys. Rev. D 90 (2014) 012013.

[9] The CMS Collaboration, CMS tracking performance results from early LHC operation, Phys. J. C 70 (2010) 1165.

[10] The LHCb Collaboration, Observation of $\mathrm{J} / \psi \mathrm{p}$ resonances consistent with pentaquark states in $\Lambda_{\mathrm{b}}^{0} \rightarrow \mathrm{J} / \psi \mathrm{pK}^{-}$decays, Phys. Rev. Lett. 115 (2015) 072001. 